\title{
Division fields of elliptic curves with minimal ramification
}

\author{
Álvaro Lozano-Robledo
}

\begin{abstract}
Let $E$ be an elliptic curve defined over $\mathbb{Q}$, let $p$ be a prime number, and let $n \geq 1$. It is well-known that the $p^{n}$-th division field $\mathbb{Q}\left(E\left[p^{n}\right]\right)$ of the elliptic curve $E$ contains all the $p^{n}$-th roots of unity. It follows that the Galois extension $\mathbb{Q}\left(E\left[p^{n}\right]\right) / \mathbb{Q}$ is ramified above $p$, and the ramification index $e\left(p, \mathbb{Q}\left(E\left[p^{n}\right]\right) / \mathbb{Q}\right)$ of any prime $\wp$ of $\mathbb{Q}\left(E\left[p^{n}\right]\right)$ lying above $p$ is divisible by $\varphi\left(p^{n}\right)$. The goal of this article is to construct elliptic curves $E / \mathbb{Q}$ such that $e\left(p, \mathbb{Q}\left(E\left[p^{n}\right]\right) / \mathbb{Q}\right)$ is precisely $\varphi\left(p^{n}\right)$, and such that the Galois group of $\mathbb{Q}\left(E\left[p^{n}\right]\right) / \mathbb{Q}$ is as large as possible, i.e., isomorphic to $\mathrm{GL}\left(2, \mathbb{Z} / p^{n} \mathbb{Z}\right)$.
\end{abstract}

\section{Introduction}

Let $E$ be an elliptic curve defined over $\mathbb{Q}$, let $p$ be a prime number, and let $n \geq 1$. The central object of study of this article is the number field $\mathbb{Q}\left(E\left[p^{n}\right]\right)$ that results by adjoining to $\mathbb{Q}$ the coordinates of all $p^{n}$-torsion points on $E(\overline{\mathbb{Q}})$, where $\overline{\mathbb{Q}}$ is a fixed algebraic closure of $\mathbb{Q}$. The existence of the Weil pairing ([24], III, Corollary 8.1.1) implies that $\mathbb{Q}\left(E\left[p^{n}\right]\right)$ contains all the $p^{n}$-th roots of unity of $\overline{\mathbb{Q}}$, i.e., we have an inclusion $\mathbb{Q}\left(\zeta_{p^{n}}\right) \subseteq \mathbb{Q}\left(E\left[p^{n}\right]\right)$, where $\zeta_{p^{n}}$ is a primitive $p^{n}$-th root of unity. It follows that the Galois extension $\mathbb{Q}\left(E\left[p^{n}\right]\right) / \mathbb{Q}$ is ramified above $p$, and the ramification index of any prime $\wp$ of $\mathbb{Q}\left(E\left[p^{n}\right]\right)$ lying above $p$, denoted by $e\left(p, \mathbb{Q}\left(E\left[p^{n}\right]\right) / \mathbb{Q}\right)$, is divisible by $\varphi\left(p^{n}\right)=e\left(p, \mathbb{Q}\left(\zeta_{p^{n}}\right) / \mathbb{Q}\right)$. The goal of this article is to construct elliptic curves $E / \mathbb{Q}$ such that $e\left(p, \mathbb{Q}\left(E\left[p^{n}\right]\right) / \mathbb{Q}\right)$ is precisely $\varphi\left(p^{n}\right)$. In other words, we are interested in finding elliptic curves such that the ramification index of the primes above $p$ in $\mathbb{Q}\left(E\left[p^{n}\right]\right) / \mathbb{Q}$ is minimal (and equal to $\varphi\left(p^{n}\right)$ ). One such example is the curve $E / \mathbb{Q}$ with Cremona label "11a1" and Weierstrass model

$$
E / \mathbb{Q}: y^{2}+y=x^{3}-x^{2}-10 x-20 .
$$

In this case $\mathbb{Q}(E[5]) / \mathbb{Q}$ is rather small; in fact, $\mathbb{Q}(E[5])=\mathbb{Q}\left(\zeta_{5}\right)$ and the ramification at 5 is indeed minimal as defined above (we will discuss this example

Mathematics Subject Classification (2010): Primary 11G05; Secondary 14H52.

Keywords: Elliptic curve, ramification, division field, torsion subgroup. 
further in Question 4.7 and Example 8.4). Moreover, we know that in general $\operatorname{Gal}\left(\mathbb{Q}\left(E\left[p^{n}\right]\right) / \mathbb{Q}\right)$ is isomorphic to a subgroup of $\operatorname{GL}\left(2, \mathbb{Z} / p^{n} \mathbb{Z}\right)$, so we are interested in constructing elliptic curves such that the extension $\mathbb{Q}\left(E\left[p^{n}\right]\right) / \mathbb{Q}$ has minimal ramification above $p$, and it is as large as possible, i.e., $\operatorname{Gal}\left(\mathbb{Q}\left(E\left[p^{n}\right]\right) / \mathbb{Q}\right) \cong$ $\operatorname{GL}\left(2, \mathbb{Z} / p^{n} \mathbb{Z}\right)$. When this occurs, we have $\operatorname{Gal}\left(\mathbb{Q}\left(E\left[p^{n}\right]\right) / \mathbb{Q}\left(\zeta_{p^{n}}\right)\right) \cong \operatorname{SL}\left(2, \mathbb{Z} / p^{n} \mathbb{Z}\right)$, and the extension $\mathbb{Q}\left(E\left[p^{n}\right]\right) / \mathbb{Q}\left(\zeta_{p^{n}}\right)$ is unramified at all primes above the rational prime $p$. For instance, this is the case for $p=5, n=5$, and the curve

$$
E / \mathbb{Q}: y^{2}+y=x^{3}-11 x+14 \text {. }
$$

Moreover, the extension $\mathbb{Q}\left(E\left[5^{5}\right]\right) / \mathbb{Q}\left(\zeta_{5^{5}}\right)$ is only ramified at primes above 2539 . The main theorem of this article is as follows.

Theorem 1.1. For every prime $p$ and every integer $n \geq 1$, and for every ordinary $j$-invariant $\lambda \in \mathbb{F}_{p}$, with $\lambda \not \equiv 0,1728 \bmod p$, there are infinitely many non-isomorphic, non-CM, elliptic curves $E$, defined over $\mathbb{Q}$, such that

(a) $j(E) \equiv \lambda \bmod p$ and $E / \mathbb{Q}$ has ordinary good reduction at $p$,

(b) the ramification index of $p$ in the extension $\mathbb{Q}\left(E\left[p^{n}\right]\right) / \mathbb{Q}$ is exactly $\varphi\left(p^{n}\right)$, and

(c) $E[p]$ an irreducible $\operatorname{Gal}(\overline{\mathbb{Q}} / \mathbb{Q})$-module.

Moreover, if $p \geq 17$ and $E$ is such an elliptic curve, the representation $\rho_{E, p^{n}}$ : $\operatorname{Gal}(\overline{\mathbb{Q}} / \mathbb{Q}) \rightarrow \operatorname{Aut}\left(E\left[p^{n}\right]\right) \cong \operatorname{GL}\left(2, \mathbb{Z} / p^{n} \mathbb{Z}\right)$ given by the action of $\operatorname{Gal}(\overline{\mathbb{Q}} / \mathbb{Q})$ on $E\left[p^{n}\right]$ is surjective. In particular, $\mathbb{Q}\left(E\left[p^{n}\right]\right) / \mathbb{Q}\left(\zeta_{p^{n}}\right)$ is a $\mathrm{SL}\left(2, \mathbb{Z} / p^{n} \mathbb{Z}\right)$ extension, unramified at primes above $p$.

For example, let $p=37$ and $n=2$. Then, for each integer $k \geq 1$, the elliptic curve

$$
E_{2, k}: y^{2}+\beta_{k} x y=x^{3}-36 \beta_{k}^{3} x-\beta_{k}^{5},
$$

with $\beta_{k}=9490+50653 k$ and $j\left(E_{2, k}\right)=11218+50653 k$, satisfies that the extension $\mathbb{Q}\left(E_{2, k}\left[37^{2}\right]\right) / \mathbb{Q}\left(\zeta_{37^{2}}\right)$ has Galois group $\operatorname{SL}\left(2, \mathbb{Z} / 37^{2} \mathbb{Z}\right)$, and it is unramified at primes above 37 (see Examples 6.4 and 7.5 for similar infinite families of elliptic curves, for any $n \geq 1$ ).

The proof of Theorem 1.1 is as follows. The existence of infinitely many elliptic curves with minimal ramification is a consequence of Gross' work on companion forms ([6]; see Section 4), the classification of non-cuspidal rational points on the modular curves $X_{0}(N)$ (see for instance Section 9 of [14]), and Hilbert's irreducibility theorem (Section 5 ). The existence of infinitely many $\operatorname{SL}\left(2, \mathbb{Z} / p^{n} \mathbb{Z}\right)$ extensions unramified above $p$ is shown in Section 7 as an application of Serre's classification of maximal subgroups of $\mathrm{GL}(2, \mathbb{Z} / p \mathbb{Z})$ as in Theorem 7.2 , and recent work of Bilu, Parent, and Rebolledo ([1], [2]) on the split case of Serre's uniformity question.

The first two sections discuss background material on Borel subgroups, and elliptic curves with ordinary good reduction, respectively. In order to apply Gross' criterion we need to calculate certain canonical lifts of $j$-invariants mod $p$. We explain how to do this in Section 4, and offer several examples (see Example 4.6). In Section 6 we provide examples of curves with minimal ramification for small primes $p$. In Section 8, we use the level 1 case of Serre's modularity conjecture [21] 
(now a theorem of Khare [9], and shown independently by Dieulefait [5]) to show the following theorem (the usual $p$-adic valuation of $\mathbb{Q}$ will be denoted by $\nu_{p}$ ).

Theorem 1.2. Let $p$ be a prime, let $n \geq 1$, and let $E / \mathbb{Q}$ be an elliptic curve such that the Galois representation on the p-torsion $\bar{\rho}_{E}: \operatorname{Gal}(\overline{\mathbb{Q}} / \mathbb{Q}) \rightarrow \mathrm{GL}(E[p])$ is absolutely irreducible, and with either good reduction at $p$, or with multiplicative reduction at $p$ and $\nu_{p}(j(E))$ divisible by $p$. Then, the extension $\mathbb{Q}\left(E\left[p^{n}\right]\right) / \mathbb{Q}\left(\zeta_{p^{n}}\right)$ is always ramified at least at one prime ideal above a rational prime $q$ distinct from $p$.

As a corollary of Theorem 1.2 , we see that any elliptic curve $E / \mathbb{Q}$ with good reduction at $p$, and such that the extension $\mathbb{Q}\left(E\left[p^{n}\right]\right) / \mathbb{Q}$ has Galois group isomorphic to $\mathrm{GL}\left(2, \mathbb{Z} / p^{n} \mathbb{Z}\right)$ and is minimally ramified at primes above $p$ (i.e., elliptic curves whose existence we prove in Theorem 1.1$)$, must also satisfy that $\mathbb{Q}\left(E\left[p^{n}\right]\right) / \mathbb{Q}\left(\zeta_{p^{n}}\right)$ ramifies at least at one prime not above $p$.

Finally, at the end of Section 8 we calculate several examples of elliptic curves with Galois group $\operatorname{Gal}\left(\mathbb{Q}\left(E\left[p^{n}\right]\right) / \mathbb{Q}\left(\zeta_{p^{n}}\right)\right)$ isomorphic to $\operatorname{SL}(2, \mathbb{Z} / p \mathbb{Z})$, and such that $\mathbb{Q}\left(E\left[p^{n}\right]\right) / \mathbb{Q}\left(\zeta_{p^{n}}\right)$ is ramified above exactly one prime $q \neq p$.

Acknowledgements. I would like to thank Kevin Buzzard, Fred Diamond, Benedict Gross, and Felipe Voloch for their help, and suggestions. I would like to give special thanks to Robert Pollack for pointing out that one can use Serre's conjecture to prove Theorem 1.2. Finally, I'd like to thank the two referees for their very helpful comments and suggestions.

\section{Borel subgroups}

In this section we discuss generalities on Borel subgroups.

Definition 2.1. Let $p$ be a prime, and $n \geq 1$. We say that a $\operatorname{subgroup~} B$ of $\mathrm{GL}\left(2, \mathbb{Z} / p^{n} \mathbb{Z}\right)$ is Borel if every matrix in $B$ is upper triangular, i.e.,

$$
B \leq\left\{\left(\begin{array}{ll}
a & b \\
0 & c
\end{array}\right): a, b, c \in \mathbb{Z} / p^{n} \mathbb{Z}, a, c \in\left(\mathbb{Z} / p^{n} \mathbb{Z}\right)^{\times}\right\}
$$

We say that $B$ is a non-diagonal Borel subgroup if none of the conjugates of $B$ in $\mathrm{GL}\left(2, \mathbb{Z} / p^{n} \mathbb{Z}\right)$ is formed solely by diagonal matrices. If $B$ is a Borel subgroup, we denote by $B_{1}$ the subgroup of $B$ formed by those matrices in $B$ whose diagonal coordinates are $1 \bmod p^{n}$, and we denote by $B_{d}$ the subgroup of $B$ formed by diagonal matrices, i.e.,

$$
B_{1}=B \cap\left\{\left(\begin{array}{ll}
1 & b \\
0 & 1
\end{array}\right): b \in \mathbb{Z} / p^{n} \mathbb{Z}\right\}, \quad B_{d}=B \cap\left\{\left(\begin{array}{ll}
a & 0 \\
0 & c
\end{array}\right): a, c \in\left(\mathbb{Z} / p^{n} \mathbb{Z}\right)^{\times}\right\}
$$

Lemma 2.2. Let $p>2$ be a prime, $n \geq 1$ and let $B \leq \mathrm{GL}\left(2, \mathbb{Z} / p^{n} \mathbb{Z}\right)$ be a Borel subgroup, such that $B$ contains a matrix $g=\left(\begin{array}{ll}a & b \\ 0 & c\end{array}\right)$ with $a \not \equiv c \bmod p$. Let $B^{\prime}=h^{-1}$ Bh with $h=\left(\begin{array}{cc}1 & b /(c-a) \\ 0 & 1\end{array}\right)$. Then, $B^{\prime} \leq \mathrm{GL}\left(2, \mathbb{Z} / p^{n} \mathbb{Z}\right)$ is a Borel subgroup conjugated to $B$ satisfying the following properties: 
(1) $B^{\prime}=B_{d}^{\prime} B_{1}^{\prime}$, i.e., for every $M \in B^{\prime}$ there is $U \in B_{d}^{\prime}$ and $V \in B_{1}^{\prime}$ such that $M=U V$; and

(2) $B /[B, B] \cong B^{\prime} /\left[B^{\prime}, B^{\prime}\right]$ and $\left[B^{\prime}, B^{\prime}\right]=B_{1}^{\prime}$.

If follows that $[B, B]=B_{1}$, and that $B_{1}$ is a cyclic subgroup of order $p^{s}$ for some $0 \leq s \leq n$.

Proof. Notice that $h^{-1} g h=\left(\begin{array}{ll}a & 0 \\ 0 & c\end{array}\right) \in B^{\prime}$. If $B^{\prime}$ only contains diagonal matrices, then $B^{\prime}=B_{d}^{\prime}$ and the statement is trivial. Otherwise, let $v\left(B^{\prime}\right)$ be the smallest nonnegative among all the top-right coordinates of matrices in $B^{\prime}$, and let $\left(\begin{array}{ll}e & f \\ 0 & l\end{array}\right) \in B^{\prime}$ such that $f \not \equiv 0 \bmod p^{n}$ and the valuation of $f$ is precisely $v\left(B^{\prime}\right)$. Then, the following commutator belongs to $B^{\prime}$ :

$$
k=\left(\begin{array}{ll}
a & 0 \\
0 & c
\end{array}\right)\left(\begin{array}{ll}
e & f \\
0 & l
\end{array}\right)\left(\begin{array}{ll}
a & 0 \\
0 & c
\end{array}\right)^{-1}\left(\begin{array}{ll}
e & f \\
0 & l
\end{array}\right)^{-1}=\left(\begin{array}{cc}
1 & \frac{f}{l}\left(\frac{a}{c}-1\right) \\
0 & 1
\end{array}\right) .
$$

Since $e, l$ are units and $a \not \equiv c \bmod p$, we conclude that $f(a / c-1) / l$ also has valuation $v\left(B^{\prime}\right)$. Let $m \in \mathbb{Z}$ be an integer such that $(f(a / c-1) / l) \cdot m \equiv p^{v\left(B^{\prime}\right)} \bmod p^{n}$. Then, $k^{m}=\left(\begin{array}{cc}1 & p^{v\left(B^{\prime}\right)} \\ 0 & 1\end{array}\right) \in B^{\prime}$. Now, if $\beta \equiv 0 \bmod p^{v\left(B^{\prime}\right)}$, then there is some $\beta^{\prime}$ such that $\beta \equiv \beta^{\prime} p^{v(B)} \bmod p^{n}$. Thus, if $M=\left(\begin{array}{ll}\alpha & \beta \\ 0 & \gamma\end{array}\right)$ is an arbitrary non-diagonal element of $B^{\prime}$, we have

$$
\begin{aligned}
M=\left(\begin{array}{ll}
\alpha & \beta \\
0 & \gamma
\end{array}\right) & =\left(\begin{array}{ll}
\alpha & \beta \\
0 & \gamma
\end{array}\right)\left(k^{m}\right)^{-\beta^{\prime} / \alpha}\left(k^{m}\right)^{\beta^{\prime} / \alpha} \\
& =\left(\begin{array}{ll}
\alpha & \beta \\
0 & \gamma
\end{array}\right)\left(\begin{array}{cc}
1 & -\frac{\beta^{\prime} p^{\nu\left(B^{\prime}\right)}}{\alpha} \\
0 & 1
\end{array}\right)\left(k^{m}\right)^{\beta^{\prime} / \alpha}=\left(\begin{array}{cc}
\alpha & 0 \\
0 & \gamma
\end{array}\right)\left(k^{m}\right)^{\beta^{\prime} / \alpha}
\end{aligned}
$$

Thus, we have shown that with $U=M\left(k^{m}\right)^{-\beta^{\prime} / \alpha} \in B_{d}^{\prime}, V=\left(k^{m}\right)^{\beta^{\prime} / \alpha} \in B_{1}^{\prime}$ we have $M=U V \in B_{d}^{\prime} B_{1}^{\prime}$. This shows (1). Moreover, it is clear that any commutator in $\left[B^{\prime}, B^{\prime}\right]$ has diagonal coordinates congruent to 1 modulo $p^{n}$ and, therefore, $\left[B^{\prime}, B^{\prime}\right] \leq B_{1}^{\prime}$. Notice that if $M \in B_{1}^{\prime}$, i.e., $\alpha \equiv \gamma \equiv 1 \bmod p^{n}$, and $m \in \mathbb{Z}$ as above, then $U$ is the identity and $M=V=\left(k^{m}\right)^{\beta^{\prime}} \in B_{1}^{\prime}$. Since $k$ is a commutator, this shows that $B_{1}^{\prime} \leq\left[B^{\prime}, B^{\prime}\right]$. Thus, $\left[B^{\prime}, B^{\prime}\right]=B_{1}^{\prime}$. Notice that $B_{1}=h B_{1}^{\prime} h^{-1}$. Hence, $[B, B]=h\left[B^{\prime}, B^{\prime}\right] h^{-1}=h B_{1}^{\prime} h^{-1}=B_{1}$, as claimed in (2). Finally, $B \cong B^{\prime}$, so

$$
B /[B, B] \cong B^{\prime} /\left[B^{\prime}, B^{\prime}\right] \cong\left(B_{d}^{\prime} B_{1}^{\prime}\right) / B_{1}^{\prime} \cong B_{d}^{\prime} \leq\left(\mathbb{Z} / p^{n} \mathbb{Z}\right)^{\times} \times\left(\mathbb{Z} / p^{n} \mathbb{Z}\right)^{\times} .
$$

This shows (2) and concludes the proof of the lemma.

Remark 2.3. The result of the previous lemma is simply false for $p=2$, i.e., the assumption $p>2$ is not just technical (the requirement $p>2$ is needed for the existence of a matrix $g$ as in the statement of the lemma). For instance, the Borel group

$$
B=\left\{\left(\begin{array}{ll}
a & b \\
0 & c
\end{array}\right): a, c \in(\mathbb{Z} / 4 \mathbb{Z})^{\times}, b \equiv 0 \bmod 2\right\} \leq \mathrm{GL}(2, \mathbb{Z} / 4 \mathbb{Z})
$$

is abelian, so the commutator of $B$ is trivial. The results of the lemma are also not necessarily true if the diagonal entries of each element in the Borel subgroup $B$ are 
congruent modulo $p$ (i.e., if there is no such element $g$ as in the statement of the lemma). For instance, let $B$ be the subgroup

$$
\left.B=\left\{\begin{array}{cc}
\left(1+p^{n-1}\right)^{t} & t\left(1+p^{n-1}\right)^{t-1} p^{n-1} \\
0 & \left(1+p^{n-1}\right)^{t}
\end{array}\right): t=1, \ldots, p\right\}
$$

of $\operatorname{GL}\left(2, \mathbb{Z} / p^{n} \mathbb{Z}\right)$. Suppose there is a subgroup $B^{\prime}$, which is a conjugate of $B$, such that $B^{\prime}=B_{d}^{\prime} B_{1}^{\prime}$. Since $B$ has order $p$, it follows that either $B \cong B_{d}^{\prime}$ or $B \cong B_{1}^{\prime}$. However, the matrices in $B$ are not diagonalizable, and 1 is not a common eigenvalue so neither isomorphism can hold.

\section{Ordinary good reduction}

Let $E$ be an elliptic curve defined over $\mathbb{Q}$, and let $p$ be a prime such that $E / \mathbb{Q}$ has good reduction at $p$. Let us fix an embedding $\iota: \overline{\mathbb{Q}} \hookrightarrow \overline{\mathbb{Q}}_{p}$. Via $\iota$, we may regard $E$ as defined over $\mathbb{Q}_{p}$. We fix a minimal model of $E$ over $\mathbb{Z}_{p}$ with good reduction, given by

$$
y^{2}+a_{1} x y+a_{3} y=x^{3}+a_{2} x^{2}+a_{4} x+a_{6},
$$

with $a_{i} \in \mathbb{Z} \subseteq \mathbb{Z}_{p}$. In particular, the discriminant $\Delta$ is a unit in $\mathbb{Z}_{p}$. Moreover, since $E / \mathbb{Z}_{p}$ has good reduction, we have an exact sequence

$$
0 \rightarrow X_{p^{n}} \rightarrow E\left(\overline{\mathbb{Q}}_{p}\right)\left[p^{n}\right] \rightarrow \widetilde{E}\left(\overline{\mathbb{F}}_{p}\right)\left[p^{n}\right] \rightarrow 0,
$$

where $\pi_{n}: E\left(\overline{\mathbb{Q}}_{p}\right)\left[p^{n}\right] \rightarrow \widetilde{E}\left(\overline{\mathbb{F}}_{p}\right)\left[p^{n}\right]$ is the homomorphism given by reduction modulo the maximal ideal of the ring of integers of $\overline{\mathbb{Q}}_{p}$, and $X_{p^{n}}$ is the kernel of $\pi_{n}$ (see [24], Ch. VII, Prop. 2.1).

From now on we assume that $E$ has ordinary good reduction at a fixed prime $p$, i.e., the reduction of $E \bmod p$, denoted by $\widetilde{E} / \mathbb{F}_{p}$, is an elliptic curve and its Hasse invariant is non-zero. It follows that $X_{p^{n}}$ and $\widetilde{E}\left(\overline{\mathbb{F}}_{p}\right)\left[p^{n}\right]$ are groups with $p^{n}$ elements ([24], Ch. V, Thm. 3.1). The Galois group $G_{p}=\operatorname{Gal}\left(\overline{\mathbb{Q}}_{p} / \mathbb{Q}_{p}\right)$ fixes $X_{p^{n}}$. If we fix a $\mathbb{Z} / p^{n} \mathbb{Z}$-basis $\left\{P_{n}, Q_{n}\right\}$ of $E\left(\overline{\mathbb{Q}}_{p}\right)\left[p^{n}\right]$, such that $X_{p^{n}}=\left\langle P_{n}\right\rangle$, then $D_{n}=$ $D_{p, n}$, the image of $G_{p}$ in $\operatorname{Aut}\left(E\left[p^{n}\right]\right)=\mathrm{GL}\left(2, \mathbb{Z} / p^{n} \mathbb{Z}\right)$, is a Borel subgroup, i.e.,

$$
D_{n} \leq\left\{\left(\begin{array}{ll}
* & * \\
0 & *
\end{array}\right)\right\} \text {. }
$$

Let $I \leq G_{p}$ be the inertia subgroup and let $I_{n}=I_{p, n}$ be the image of $I$ in $\operatorname{Aut}\left(E\left[p^{n}\right]\right) \cong \operatorname{GL}\left(2, \mathbb{Z} / p^{n} \mathbb{Z}\right)$. Then, $I$ acts on $\widetilde{E}(\overline{\mathbb{F}})\left[p^{n}\right]$ trivially (because $I$ acts trivially on the residue field; see [24], Ch. VII, §4, or [18], Prop. 11, for details in the case when $n=1)$, and therefore $I$ acts on $X_{p^{n}}$ via $\chi_{n}: G_{p} \rightarrow\left(\mathbb{Z} / p^{n} \mathbb{Z}\right)^{\times}$, the cyclotomic character modulo $p^{n}$, because the determinant of $\rho_{E, p^{n}}: G_{p} \rightarrow$ $\operatorname{Aut}\left(E\left[p^{n}\right]\right) \cong \mathrm{GL}\left(2, \mathbb{Z} / p^{n} \mathbb{Z}\right)$ is precisely $\chi_{n}$. Thus,

$$
I_{n} \leq\left\{\left(\begin{array}{cc}
\chi_{n} & * \\
0 & 1
\end{array}\right)\right\}
$$

In what follows, we fix a prime $\bar{\Omega}$ of $\overline{\mathbb{Q}}$ over $p$, and let $\iota: \overline{\mathbb{Q}} \hookrightarrow \overline{\mathbb{Q}}_{p}$ be the embedding associated to $\bar{\Omega}$. Via $\iota$, we may consider an elliptic curve $E / \mathbb{Q}$ as an elliptic curve 
defined over $\mathbb{Q}_{p}$. Let $\Omega$ be a prime of $\mathbb{Q}\left(E\left[p^{n}\right]\right)$ lying under $\bar{\Omega}$, and let $D_{\Omega, n}$ and $I_{\Omega, n}$ be respectively the decomposition and inertia subgroups of $\operatorname{Gal}\left(\mathbb{Q}\left(E\left[p^{n}\right]\right) / \mathbb{Q}\right)$ associated to $\Omega$. In this setting $D_{n}$, as above, can be identified with $D_{\Omega, n}$, and $I_{n}$ is identified with $I_{\Omega, n}$.

Lemma 3.1. If the decomposition group $D_{n}$ is diagonalizable, then inertia $I_{n}$ is diagonalizable. If $p>2$, the converse is also true.

Proof. One direction is trivial: if $D_{n}$ is diagonalizable, then $I_{n} \leq D_{n}$ must be diagonalizable as well. Let us now suppose now that $p>2$. By our remarks above, the decomposition group $D_{n}$ is a Borel. Let us assume that $I_{n}$ is diagonal, i.e., $I_{n}=\left\{\left(\begin{array}{cc}\chi_{n} & 0 \\ 0 & 1\end{array}\right)\right\}$, with respect to a $\mathbb{Z} / p^{n} \mathbb{Z}$-basis $\left\{P_{n}, Q_{n}\right\}$ of $E\left[p^{n}\right]$. Let $D_{n, 1}$ and $D_{n, d}$ be the subgroups of $D_{n}$ defined as in Definition 2.1. Since $p>2$ and since the cyclotomic character $\chi_{n}: I_{n} \rightarrow\left(\mathbb{Z} / p^{n} \mathbb{Z}\right)^{\times}$is surjective (the base field here is $\left.\mathbb{Q}_{p}\right)$, there is a diagonal matrix $M=\left(\begin{array}{ll}a & 0 \\ 0 & 1\end{array}\right)$ in $I_{n} \leq D_{n}$ with $a \not \equiv 1 \bmod p$. Hence, we can apply Lemma 2.2 with $B=D_{n}, g=M$, and $h=\mathrm{Id}$, and so $B=B^{\prime}=D_{n}=D_{n, d} D_{n, 1}$. Let $L$ be the subfield of $\mathbb{Q}_{p}\left(E\left[p^{n}\right]\right)$ fixed by $D_{n, 1}$. It follows that $\operatorname{Gal}\left(\mathbb{Q}_{p}\left(E\left[p^{n}\right]\right) / L\right) \cong D_{n, 1}$, so the extension is cyclic, of degree $p^{s}$, for some $0 \leq s \leq n$, and it is unramified because $I_{n} \cap D_{n, 1}=\{\operatorname{Id}\}$. Then, $\mathbb{Q}_{p}\left(E\left[p^{n}\right]\right) / L$ is a finite unramified extension and, therefore, it is generated by a root of unity $\zeta$ of prime-to- $p$ order $([8]$, p. 37$)$. But, in this case, $\mathbb{Q}_{p}(E[p])=L(\zeta)$ would be abelian over $\mathbb{Q}_{p}$. Since $\operatorname{Gal}\left(\mathbb{Q}_{p}(E[p]) / \mathbb{Q}_{p}\right) \cong D_{n}$ and $D_{n, 1}$ is the commutator subgroup of $D_{n}$, this is only possible if $D_{n, 1}$ is trivial and $D_{n}=D_{n, d}$ is diagonalizable.

Remark 3.2. The converse part of Lemma 3.1 (i.e., if $p>2$ and $I_{n}$ is diagonalizable, then $D_{n}$ is diagonalizable) is not used in the proof of our results, but we have included it here as it is interesting in itself.

Remark 3.3. The converse of the previous lemma is false for $p=2$. For instance, let $E$ be the curve with Cremona label "15a2", given by the model $y^{2}+x y+y=$ $x^{3}+x^{2}-135 x-660$. The curve $E$ has ordinary good reduction at $p=2$. The 2-torsion of $E$ is rational, so $\mathbb{Q}(E[2]) / \mathbb{Q}$ is trivial and, therefore, $\mathbb{Q}_{2}(E[2]) / \mathbb{Q}_{2}$ is trivial as well. Thus, $D_{1} \cong \operatorname{Gal}\left(\mathbb{Q}_{2}(E[2]) / \mathbb{Q}_{2}\right)$ and $I_{1}$ are trivially diagonalizable. However, even though $D_{2}$ is not, $I_{2}$ is diagonalizable.

The extension $\mathbb{Q}(E[4]) / \mathbb{Q}$ is of degree 4 , isomorphic to a subgroup of the linear group $\mathrm{GL}(2, \mathbb{Z} / 4 \mathbb{Z})$ of the form

$$
B=\left\{\left(\begin{array}{ll}
a & b \\
0 & 1
\end{array}\right): a \in(\mathbb{Z} / 4 \mathbb{Z})^{\times}, b \equiv 0 \bmod 2\right\} \cong \mathbb{Z} / 2 \mathbb{Z} \times \mathbb{Z} / 2 \mathbb{Z} .
$$

Thus, $K=\mathbb{Q}(E[4])$ is abelian over $\mathbb{Q}$ (see Remark 2.3) and, in fact, $K=\mathbb{Q}(i, \sqrt{5})$. Let $\mathcal{O}$ be the maximal order in $K=\mathbb{Q}(E[4])$. Since 2 remains prime in $\mathbb{Q}(\sqrt{5})$ and it ramifies in $\mathbb{Q}(i)$, it follows that $2 \mathcal{O}=\wp^{2}$ is the square of a prime ideal $\wp$ of $K$ above 2. Hence, $\mathbb{Q}_{2}(E[4]) / \mathbb{Q}_{2}$ is also an extension of degree 4 (a ramified extension of degree 2 followed by an unramified extension also of degree 2), with Galois group $D_{2} \cong B$ which is not diagonalizable. However, $I_{2} \cong\left\{\left(\begin{array}{ll}a & 0 \\ 0 & 1\end{array}\right): a \in(\mathbb{Z} / 4 \mathbb{Z})^{\times}\right\}$ is diagonalizable. 
Results of Serre ([22], A.2.4) and Lemma 3.1 show that, for $p>2$, the inertia subgroup $I_{n}$ is not diagonalizable for all $n \geq 1$ if and only if $E$ is not a CM curve. We obtain:

Theorem 3.4. Let $p>2$. The following statements are equivalent:

(1) The elliptic curve $E$ has $C M$ (over an extension of $\mathbb{Q}_{p}$ ).

(2) The exact sequence

$$
0 \rightarrow X \rightarrow V_{p}(E) \rightarrow V_{p}(\widetilde{E}) \rightarrow 0
$$

is split, where $X=\left(\lim _{\longleftarrow} X_{p^{n}}\right) \otimes \mathbb{Q}_{p}$, and $V_{p}(E)=T_{p}(E) \otimes \mathbb{Q}_{p}$.

(3) The decomposition subgroups $D_{n} \cong \operatorname{Gal}\left(\mathbb{Q}_{p}\left(E\left[p^{n}\right]\right) / \mathbb{Q}_{p}\right)$ are diagonalizable for all $n \geq 1$.

(4) The inertia subgroups $I_{n}$ are diagonalizable for all $n \geq 1$.

Proof. The equivalence of (1), (2), and (3) is due to Serre. The equivalence between (3) and (4) follows from Lemma 3.1.

Lemma 3.5. Let $p>2$ be a prime. Let $E / \mathbb{Q}$ be an elliptic curve with ordinary good reduction at $p$. With notation as above, suppose that $I_{m}$ is diagonalizable but $I_{m+1}$ is not, for some $m \geq 1$ (or $m=\infty$ if $E$ has $C M$ ). Then there is a $\mathbb{Z}_{p}$-basis $\mathcal{B}$ of $T_{p}(E)$ such that the image of inertia, $I$, has the following structure:

$$
I=\left\{\left(\begin{array}{cc}
\chi & b \\
0 & 1
\end{array}\right): b \equiv 0 \bmod p^{m}\right\} \leq \mathrm{GL}\left(2, \mathbb{Z}_{p}\right)
$$

where $\chi: \operatorname{Gal}\left(\overline{\mathbb{Q}}_{p} / \mathbb{Q}_{p}\right) \rightarrow \mathbb{Z}_{p}^{\times}$is the cyclotomic character.

Proof. By the remarks at the beginning of this section, we know that each $I_{n}$ and

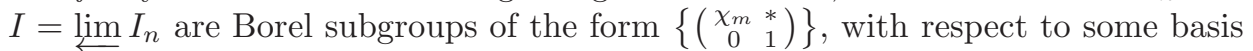
$\left\{P_{n}, \overleftarrow{Q_{n}}\right\}$ of $E\left[p^{n}\right]$, respectively, where $\chi_{m}$ is the reduction of $\chi$ modulo $p^{m}$. Since $p>2$ and $\chi_{m}$ is surjective, Lemma 2.2 implies the existence of a basis $\left\{P_{n}, Q_{n}^{\prime}\right\}$ of $E\left[p^{n}\right]$ such that $I_{n}=\left(I_{n}\right)_{d} \cdot\left(I_{n}\right)_{1}$, where

$$
\left(I_{n}\right)_{d}=\left\{\left(\begin{array}{cc}
\chi_{m} & 0 \\
0 & 1
\end{array}\right)\right\}, \text { and }\left(I_{n}\right)_{1}=I_{n} \cap\left\{\left(\begin{array}{cc}
1 & * \\
0 & 1
\end{array}\right)\right\}
$$

Hence, if we put $P=\left(P_{n}\right)_{n=1}^{\infty}$ and $Q^{\prime}=\left(Q_{n}^{\prime}\right)_{n=1}^{\infty} \in T_{p}(E)$, then $\left\{P, Q^{\prime}\right\}$ is a $\mathbb{Z}_{p}$-basis of $T_{p}(E)$ such that $I=(I)_{d} \cdot(I)_{1}$, where

$$
(I)_{d}=\left\{\left(\begin{array}{cc}
\chi & 0 \\
0 & 1
\end{array}\right)\right\}, \text { and }(I)_{1}=I \cap\left\{\left(\begin{array}{cc}
1 & * \\
0 & 1
\end{array}\right)\right\} .
$$

Since $(I)_{1}$ is an abelian subgroup of $I$, the top right coordinates of the matrices in $(I)_{1}$ form an additive subgroup $H$ of $\mathbb{Z}_{p}$, say $H=p^{t} \mathbb{Z}_{p}$ for some $t \geq 0$. Thus,

$$
(I)_{1}=\left\{\left(\begin{array}{ll}
1 & b \\
0 & 1
\end{array}\right): b \in p^{t} \mathbb{Z}_{p}\right\}
$$


First, suppose that $m$ is finite. Since $I_{m} \equiv I \bmod p^{m}$ is diagonalizable, we must have $t \geq m$, and since $I_{m+1}$ is not diagonalizable, it follows $t=m$. This shows that

$$
I=\left\{\left(\begin{array}{cc}
\chi & b \\
0 & 1
\end{array}\right): b \equiv 0 \bmod p^{m}\right\} \leq \mathrm{GL}\left(2, \mathbb{Z}_{p}\right)
$$

as desired. If $m=\infty$, then $t$ must be arbitrarily large, and so $b \in(0)$.

The structure of the inertia subgroup described in the previous lemma has the following corollary on ramification indices.

Theorem 3.6. Let $E / \mathbb{Q}$ be an elliptic curve without $C M$, and with ordinary good reduction at a prime $p$. If $I_{m}$ is diagonalizable for some integer $m \geq 1$, then so is $I_{n}$ for all integers $1 \leq n \leq m$. Moreover, if $p>2$ and $m$ is the largest integer such that $I_{m}$ is diagonalizable, then the ramification index of $p$ in the extension $\mathbb{Q}\left(E\left[p^{n}\right]\right) / \mathbb{Q}$ is given by $\varphi\left(p^{n}\right)$ if $1 \leq n \leq m$, and by $\varphi\left(p^{n}\right) \cdot p^{n-m}$ if $n>m$.

Proof. Let $\Omega$ be a fixed prime of $\mathbb{Q}\left(E\left[p^{n}\right]\right)$ above $p$. Suppose that there exists an integer $n$ such that $I_{n}=I_{n}(\Omega \mid p)$ is diagonalizable, and let $m$ be the largest such integer (a largest $m$ exists by Theorem 3.4 because $E$ does not have CM). Then, for every $1 \leq n \leq m$, there is a basis of $E\left[p^{n}\right]$ such that

$$
I_{n}(\Omega \mid p) \cong\left\{\left(\begin{array}{cc}
\chi & 0 \\
0 & 1
\end{array}\right)\right\} \cong\left\{\left(\begin{array}{cc}
a & 0 \\
0 & 1
\end{array}\right): a \in\left(\mathbb{Z} / p^{n} \mathbb{Z}\right)^{\times}\right\} \leq \mathrm{GL}\left(2, \mathbb{Z} / p^{n} \mathbb{Z}\right)
$$

where $\chi: \operatorname{Gal}(\overline{\mathbb{Q}} / \mathbb{Q}) \rightarrow\left(\mathbb{Z} / p^{n} \mathbb{Z}\right)^{\times}$is the cyclotomic character (which is surjective). Since $\mathbb{Q}\left(E\left[p^{n}\right]\right) / \mathbb{Q}$ is Galois, the ramification index of any prime of $\mathbb{Q}\left(E\left[p^{n}\right]\right)$ over $p$ is the same, and it follows that the ramification index of $p$ in $\mathbb{Q}\left(E\left[p^{n}\right]\right) / \mathbb{Q}$ is $\varphi\left(p^{n}\right)$ if $1 \leq n \leq m$, as claimed.

If $p>2$, then Lemma 3.5 implies that the image of the inertia subgroup, $I_{n}(\Omega \mid p)$, is of the form

$$
I_{n}(\Omega \mid p)=\left\{\left(\begin{array}{ll}
a & b \\
0 & 1
\end{array}\right): a \in\left(\mathbb{Z} / p^{n} \mathbb{Z}\right)^{\times}, b \equiv 0 \bmod p^{m}\right\} \leq \mathrm{GL}\left(2, \mathbb{Z} / p^{n} \mathbb{Z}\right) .
$$

Therefore, the ramification index of $p$ in $\mathbb{Q}\left(E\left[p^{n}\right]\right) / \mathbb{Q}$ is $\varphi\left(p^{n}\right) \cdot p^{n-m}$ if $n \geq m$, as desired.

\section{Gross' criterion and canonical liftings}

We now turn our attention to finding $m$ such that $I_{m}$ is diagonalizable, but $I_{m+1}$ is not. A deep theorem of Gross provides the criterion we seek.

Theorem 4.1 (Gross; see [6], p. 514; see also $§ 14-15)$. Let $p$ be a prime, and let $E / \mathbb{Q}$ be an elliptic curve with ordinary good reduction at $p$, with $j \neq 0,1728$, and assume that $E[p]$ is an irreducible $\operatorname{Gal}(\overline{\mathbb{Q}} / \mathbb{Q})$-module. Let $D_{n} \leq \operatorname{Gal}\left(\mathbb{Q}\left(E\left[p^{n}\right]\right) / \mathbb{Q}\right) \leq$ $\mathrm{GL}\left(2, \mathbb{Z} / p^{n} \mathbb{Z}\right)$ be a decomposition group at $p$. Let $j_{E}=j(E)$ be the $j$-invariant 
of $E$ and let $j_{0}$ be the $j$-invariant of the "canonical lifting" of the reduction of $j(E)$ modulo $p$, i.e., $j_{0}$ is the $j$-invariant of the unique elliptic curve $E_{0} / \mathbb{Q}_{p}$ which satisfies $E_{0} \equiv E \bmod p$ and $\operatorname{End}_{\mathbb{Q}_{p}}\left(E_{0}\right) \equiv \operatorname{End}_{\mathbb{F}_{p}}(E)$. Then $D_{n}$ is diagonalizable if and only if $j_{E} \equiv j_{0} \bmod p^{n+1}$ if $p$ is odd, and $j_{E} \equiv j_{0} \bmod 2^{n+2}$ if $p=2$.

In order to use Gross' criterion (Theorem 4.1) we need to be able to calculate canonical liftings. In the rest of this section, we explain how to do so, and calculate a canonical lifting in several examples.

Theorem 4.2 (Deuring; see [15], §8). Let $\mathbb{F}$ be a perfect field of characteristic $p>0$, and let $E$ be an elliptic curve with $j(E) \in \mathbb{F}$ and with Hasse invariant $\neq 0$ (i.e., having the maximum number of points of order $p)$. Let $T_{p}(x, y)$ be the classical modular polynomial relating the $j$-invariants of elliptic curves that have isogenies of degree $p$ between themselves. Let $W(\mathbb{F})$ be the ring of Witt vectors with coefficients in $\mathbb{F}$ and let $s: W(\mathbb{F}) \rightarrow W(\mathbb{F})$ be the Frobenius automorphism given by $\left(x_{0}, x_{1}, \ldots\right) \rightarrow\left(x_{0}^{p}, x_{1}^{p}, \ldots\right) \in W(\mathbb{F})$ in Witt vector coordinates. Then, there is a canonical lifting of $E / \mathbb{F}$ to $W(\mathbb{F})$, with $j$-invariant $j_{0} \in W(\mathbb{F})$. Moreover,

(1) one has $T_{p}\left(j_{0}, s\left(j_{0}\right)\right)=0$ and $j_{0} \equiv j(E) \bmod p$; and

(2) if $j(E) \notin \mathbb{F}_{p^{2}}$, then there is a unique solution $j_{0} \in W(\mathbb{F})$ with $T_{p}\left(j_{0}, s\left(j_{0}\right)\right)$ $=0$ and $j_{0} \equiv j(E) \bmod p$.

Corollary 4.3. Let $p$ be a prime and let $E / \mathbb{Q}$ be an elliptic curve with ordinary good reduction at $p$. Then, there is a canonical lifting of $E / \mathbb{F}_{p}$ to $\mathbb{Q}_{p}$, with $j$ invariant $j_{0} \in \mathbb{Q}_{p}$. Moreover, $j_{0}$ satisfies $T_{p}\left(j_{0}, j_{0}\right)=0$ and $j_{0} \equiv j(E) \bmod p$.

The proof of the corollary is clear, since $W\left(\mathbb{F}_{p}\right)=\mathbb{Q}_{p}$ and the Frobenius automorphism of Witt coordinates is the identity, as $x \mapsto x^{p}$ fixes $\mathbb{F}_{p}$.

Example 4.4. The classical modular polynomials $T_{p}(x, y)$ for $p=2$ and 3 are given by

$$
\begin{aligned}
T_{2}(x, y)= & x^{3}-x^{2} y^{2}+1488 x^{2} y-162000 x^{2}+1488 x y^{2}+40773375 x y \\
& +8748000000 x+y^{3}-162000 y^{2}+8748000000 y-157464000000000, \\
T_{3}(x, y)= & x^{4}-x^{3} y^{3}+2232 x^{3} y^{2}-1069956 x^{3} y \\
& +36864000 x^{3}+2232 x^{2} y^{3}+2587918086 x^{2} y^{2}+8900222976000 x^{2} y \\
& +452984832000000 x^{2}-1069956 x y^{3}+8900222976000 x y^{2} \\
& -770845966336000000 x y \\
& +1855425871872000000000 x+y^{4}+36864000 y^{3} \\
& +452984832000000 y^{2}+1855425871872000000000 y,
\end{aligned}
$$


and $T_{2}(x, x), T_{3}(x, x)$ factor as

$$
\begin{aligned}
T_{2}(x, x)= & -x^{4}+2978 x^{3}+40449375 x^{2}+17496000000 x-157464000000000 \\
= & -(x-8000)(x-1728)(x+3375)^{2} . \\
T_{3}(x, x)= & -x^{6}+4464 x^{5}+2585778176 x^{4}+17800519680000 x^{3} \\
& -769939996672000000 x^{2}+3710851743744000000000 x \\
= & -x(x-54000)(x-8000)^{2}(x+32768)^{2} .
\end{aligned}
$$

Similarly, the polynomial $T_{5}(x, x)$ factors as

$$
\begin{aligned}
T_{5}(x, x)= & (x-287496)^{2} \cdot(x-1728)^{2} \cdot(x+32768)^{2} \cdot(x+884736)^{2} \\
& \cdot\left(x^{2}-1264000 x-681472000\right) .
\end{aligned}
$$

Hence, if $E / \mathbb{Q}$ is an elliptic curve with ordinary good reduction at $p=2,3$, or 5 , then the canonical lift of the reduction of $E$ modulo $p$ is the following:

- If $p=2$ and $j(E) \equiv 1 \bmod 2$, then $j_{0}=-3375=-3^{3} \cdot 5^{3}$.

- If $p=3$, and $j(E) \equiv 1$ or $2 \bmod 3$, then $j_{0}=-32768=-2^{15}$ or $j_{0}=8000=$ $2^{6} \cdot 5^{3}$, respectively.

- If $p=5$, and $j(E) \equiv 1,2$ or $4 \bmod 5$, then $j_{0}=287496=2^{3} 3^{3} 11^{3}, j_{0}=$ $-32768=-2^{15}$, or $j_{0}=-884736=-2^{15} \cdot 3^{3}$, respectively.

Example 4.5. Let $p=37$. Let $T_{37}(x, y)$ be the classical modular polynomial, and put $f(x)=T_{37}(x, x) \in \mathbb{Z}[x]$. The degree of the polynomial $f(x)$ is 74 and it factors, over $\mathbb{Q}[x]$, as a product of 20 polynomials $f(x)=\prod_{i=1}^{20} p_{i}(x)^{m_{i}}$ of degree $d_{i}$ and multiplicity $m_{i}$, as follows:

\begin{tabular}{l|ccccccccccccccc}
\hline & 1 & 2 & 3 & 4 & 5 & 6 & 7 & 8 & 9 & 10 & $11, \ldots, 16$ & 17 & 18 & 19 & 20 \\
\hline$d_{i}$ & 1 & 1 & 1 & 1 & 1 & 1 & 1 & 1 & 1 & 2 & 2 & 3 & 4 & 4 & 4 \\
\hline$m_{i}$ & 2 & 2 & 2 & 2 & 2 & 2 & 2 & 2 & 2 & 1 & 2 & 2 & 2 & 2 & 2 \\
\hline
\end{tabular}

Notice that $f(x) \equiv-\left(x^{37}-x\right)^{2} \bmod 37$. By the chart above, $p_{10}$ is the only polynomial divisor of $f(x)$ whose multiplicity is not 2 over $\mathbb{Q}[x]$; however, $p_{10} \equiv$ $(x-8)^{2}$ is a square over $(\mathbb{Z} / 37 \mathbb{Z})[x]$. It follows that there is a unique polynomial $p_{i}(x)$, for some $1 \leq i \leq 20$, such that one of the roots $j_{0} \in \mathbb{Q}_{37}$ of $p_{i}(x)$ is congruent to $7 \bmod 37$ (and $j_{0}$ is the unique root of $f(x)$ with this property). Indeed, direct computation reveals that the only polynomial $p_{i}(x)$ with $7 \bmod 37$ as a root is $p_{20}(x)$. The polynomial $p_{20}(x)$ is given by

$$
\begin{aligned}
p_{20}(x)= & x^{4}-3196800946944 x^{3}-5663679223085309952 x^{2} \\
& +88821246589810089394176 x-5133201653210986057826304 \\
\equiv & (x-2)(x-3)(x-7)(x-28) \bmod 37 \mathbb{Z}[x],
\end{aligned}
$$

and its root $j_{0} \in \mathbb{Q}_{37}$ has the following 37-adic expansion:

$$
j_{0}=\left(7,266,11218,1632114 \bmod 37^{4}, 12877080 \bmod 37^{5}, \ldots\right) \in \mathbb{Q}_{37} .
$$


Example 4.6. In the table below, we list the first few primes $(p \leq 20)$, together with those canonical lifts $j_{0}$ that are in $\mathbb{Z}_{p}$ (and not in some extension of $\mathbb{Z}_{p}$ ). If $j_{0} \in \mathbb{Z}$, then we list the actual value of $j_{0}$ in the first line. If $j_{0} \in \mathbb{Z}_{p}$, then we list a second (and third) line of values modulo $p^{5}$.

\begin{tabular}{c|c}
\hline$p$ & Canonical lifts \\
\hline 2 & $-3375,1728,8000$ \\
\hline 3 & $-32768,0,8000,54000$ \\
\hline 5 & $-884736,-32768,1728,287496$ \\
\hline 7 & $-7598,2126+\mathrm{O}\left(7^{5}\right)$ \\
\hline 11 & $-884736000,-884736,-32768,-3375,8000,16581375$, \\
& $7665,24243,27342,35982,61340+\mathrm{O}\left(11^{5}\right)$ \\
\hline 13 & $-884736000,-12288000,0,1728,54000,287496$, \\
& $-159805,-102235,-71051,10643,33871,64521+\mathrm{O}\left(13^{5}\right)$ \\
\hline 17 & $-147197952000,-884736000,-884736,1728,8000,287496$, \\
& $-675116,-672317,-362937,-158485,-126224+\mathrm{O}\left(17^{5}\right)$, \\
& $-110190,74802,128731,229973+\mathrm{O}\left(17^{5}\right)$ \\
\hline 19 & $-147197952000,-12288000,-884736,0,8000,54000$ \\
& $-752904,-695235,-605629,-570609,-515098+\mathrm{O}\left(19^{5}\right)$, \\
& $-118930,318870,414604,526924,710891,1034963+\mathrm{O}\left(19^{5}\right)$, \\
& $1149479,1187960+\mathrm{O}\left(19^{5}\right)$. \\
\hline
\end{tabular}

Question 4.7. In Gross' criterion (Theorem 4.1), it is assumed that $E[p]$ is an irreducible Galois module. Is this hypothesis necessary? I.e., suppose $E / \mathbb{Q}$ is ordinary good at $p>2$, but $E[p]$ is reducible. Does Gross' criterion still work in this case? Indeed, we have verified that the criterion holds in many examples where $E[p]$ is reducible.

For instance, let $E / \mathbb{Q}$ be the curve with $j$-invariant $j(E)=-122023936 / 161051$ and Cremona label 11a1, given by a Weierstrass model

$$
E: y^{2}+y=x^{3}-x^{2}-10 x-20 .
$$

The curve $E$ has ordinary good reduction at $p=5$, however, there is a 5 -torsion point defined over $\mathbb{Q}$, namely $P=(16,60)$. Hence, $\rho_{E, p}$ is reducible. Nonetheless,

$$
j(E)=-122023936 / 161051 \equiv 14 \equiv-884736 \bmod 25,
$$

where $j_{0}=-884736$ is the canonical lift of $4 \in \mathbb{F}_{5}$. In particular, (if $E[5]$ was irreducible, then) Gross' criterion would imply that $\mathbb{Q}(E[5]) / \mathbb{Q}\left(\zeta_{5}\right)$ is unramified at the prime above 5 . This conclusion is indeed true because the extension is trivial, i.e., $\mathbb{Q}(E[5])=\mathbb{Q}\left(\zeta_{5}\right)$ where $E[5]$ is generated by the 5 -torsion points $P=(16,60)$ and $Q=\left(4 \zeta_{5}^{3}+2 \zeta_{5}^{2}+3 \zeta_{5}+2,3 \zeta_{5}^{3}-4 \zeta_{5}^{2}+5 \zeta_{5}\right)$.

\section{Curves with minimal ramification at $p$}

We begin with a summary of the definitions and the precise statement of Hilbert's irreducibility theorem (see [13], Chapter 9) that we will use in the proof of Theorem 5.4 (see [23], Chapter 3, for another flavor of Hilbert's irreducibility). 
Definition 5.1 ([13], Ch. 9). Let $K$ be a field of characteristic 0, and suppose that $f\left(t_{1}, \ldots, t_{r}, X_{1}, \ldots, X_{s}\right)=f(\mathbf{t}, \mathbf{X}) \in K(\mathbf{t})[\mathbf{X}]$ is a polynomial in $X_{1}, \ldots, X_{s}$ with coefficients in $K(\mathbf{t})$ which is irreducible as a polynomial in $\mathbf{X}$ variables. A basic Hilbert set is a subset $U_{f, K}$ of the affine space $\mathbb{A}^{r}(K)$ consisting of those points $\mathbf{t}^{\prime}=\left(t_{1}^{\prime}, \ldots, t_{r}^{\prime}\right) \in K^{r}$ at which the coefficients of $f$ are defined, and such that $f\left(\mathbf{t}^{\prime}, \mathbf{X}\right)$ is irreducible in $K[\mathbf{X}]$ over $K$. A Hilbert subset of $\mathbb{A}^{r}(K)$ is a set defined as the intersection of a finite number of basic Hilbert sets with a finite number of non-empty Zariski open subsets of $\mathbb{A}^{r}(K)$. A field $K$ is called hilbertian if the Hilbert subsets of $\mathbb{A}^{r}(K)$ are not empty (and thus are infinite).

Theorem 5.2 ([13], Ch. 9). A number field is hilbertian.

Theorem 5.3 ([13], Ch. 9, Corollary 2.5). A Hilbert subset of $\mathbb{Q}$ is dense for the ordinary topology and every $p$-adic topology on $\mathbb{Q}$.

We are now ready to prove the first part of Theorem 1.1.

Theorem 5.4. For every prime $p$ and every $n \geq 1$, and for every ordinary $j$ invariant $\lambda \in \mathbb{F}_{p}$, with $\lambda \not \equiv 0,1728 \bmod p$, there are infinitely many non isomorphic, non-CM, elliptic curves $E$, defined over $\mathbb{Q}$, with $j(E) \equiv \lambda \bmod p$ (and with ordinary good reduction at $p)$ such that the ramification index of $p$ in the extension $\mathbb{Q}\left(E\left[p^{n}\right]\right) / \mathbb{Q}$ is exactly $\varphi\left(p^{n}\right)$, and $E[p]$ is an irreducible $\operatorname{Gal}(\overline{\mathbb{Q}} / \mathbb{Q})$-module. In particular, $\mathbb{Q}\left(E\left[p^{n}\right]\right) / \mathbb{Q}\left(\zeta_{p^{n}}\right)$ is unramified at $p$.

Proof. Let $p$ be a fixed prime, and let $U$ be the subset of $\mathbb{Q}$ formed by those $j$ invariants $\iota_{0} \in \mathbb{Q}$ such that if $E / \mathbb{Q}$ is an elliptic curve with $j(E)=\iota_{0}$, then $E[p]$ is an irreducible $\operatorname{Gal}(\overline{\mathbb{Q}} / \mathbb{Q})$-module. We claim that $U$ contains a Hilbert set $V \subseteq U$. Indeed, if $E[p]$ is reducible, then $j(E)$ gives rise to a non-cuspidal rational point on the modular curve $X_{0}(p)$. We distinguish two cases:

- If $X_{0}(p)$ is a curve of genus $\geq 1$ and $X_{0}(p)(\mathbb{Q})$ is non-empty, then Mazur's theorem on isogenies of prime degree $([16])$ says that $p$ is a prime in the list $11,17,19,37,43,67,163$, but in all these cases $X_{0}(p)(\mathbb{Q})$ has only finitely many points (see for example Section 9 and Table 4 of [14]). Hence, there are at most finitely many exceptions in $\iota_{0} \in \mathbb{Q}$ such that $E[p]$ is reducible. Hence $V=U$ is a non-empty Zariski open set of $\mathbb{Q}$, and therefore a Hilbert set.

- If $X_{0}(p)$ is a curve of genus 0 , then the set of $j$-invariants of elliptic curves over $\mathbb{Q}$ with $E[p]$ reducible is given by a one parameter family

$$
S_{\text {red }, p}=\left\{\phi_{p}(h): h \in \mathbb{Q}\right\}
$$

where $\phi_{p}(h)$ is a rational function of degree $\geq 3$ (see [14], Section 9, for the explicit rational function $\left.\phi_{p}\right)$. Let $\phi_{p}(h)=u_{p}(h) / v_{p}(h)$, where $u_{p}$ and $v_{p}$ are relatively prime polynomials in $\mathbb{Q}[h]$. Then, $\iota_{0} \in \mathbb{Q}$ is in $U$ if and only if $\phi_{p}(h)=\iota_{0}$ has no root $h_{0} \in \mathbb{Q}$ or, equivalently, if $u_{p}(h)-\iota_{0} \cdot v_{p}(h)=0$ has no root $h_{0} \in \mathbb{Q}$. If we put $f_{p}(j, x)=u_{p}(x)-j v_{p}(x)$, then the basic Hilbert set $V=U_{f_{p}, \mathbb{Q}}$ is contained in $U$, since $\iota_{0} \in U_{f_{p}, \mathbb{Q}}$ implies that $f_{p}\left(\iota_{0}, x\right)$ is irreducible over $\mathbb{Q}$, and therefore has no rational roots $x_{0} \in \mathbb{Q}$.

Therefore, in all cases $U$ contains a Hilbert set. 
Let $\lambda \in \mathbb{F}_{p}$ be a fixed ordinary $j$-invariant, with $\lambda \not \equiv 0$ or $1728 \bmod p$. Notice that there is always at least one such ordinary $j$-invariant $\lambda$ in $\mathbb{F}_{p}$ : if $p=2$, then $\lambda=$ 1 ; if $p=3$, we may pick $\lambda \equiv 1$ or $2 \bmod 3$; if $p=5$, we may pick $\lambda \equiv 1,2$ or $4 \bmod 5$; if $p>5$, there are at least $p-\left([p / 12]+\varepsilon_{p}\right) \geq 11 p / 12-2 \geq 11 \cdot 7 / 12-2 \geq 4$ ordinary $j$ invariants in $\mathbb{F}_{p}$, where $\varepsilon_{p}=0,1,1,2$ if $p \equiv 1,5,7,11 \bmod 12$, respectively (see [24], Ch. V, Theorem 4.1.(c)), so at least one of them is $\not \equiv 0$ or $1728 \bmod p$.

Let $E_{0} / \mathbb{Q}_{p}$ be the unique canonical lift to $\mathbb{Q}_{p}$ with $j$-invariant $j_{0}=j\left(E_{0}\right) \equiv \lambda$ modulo $p$, and define

$$
C_{\lambda, n}=\left\{j \in \mathbb{Q} \cap \mathbb{Z}_{p}: j \equiv j_{0} \bmod p^{n+1}\right\}
$$

for $p>2$, and $C_{\lambda, n}=\left\{j \in \mathbb{Q} \cap \mathbb{Z}_{2}: j \equiv j_{0} \bmod 2^{n+2}\right\}$ when $p=2$. By Theorem 5.2 , the field $\mathbb{Q}$ is hilbertian and, by Theorem 5.3, the Hilbert set $V \subseteq U$ is dense for the $p$-adic topology. Since $C_{\lambda, n}$ is an open set $p$-adically, it follows that $C_{\lambda, n} \cap V$ is infinite and contained in $U$. Moreover, there are only 13 rational $\mathrm{CM} j$-invariants (see [25], Appendix A, §3). Hence, the set $H_{\lambda, n}$ of $j$-invariants with $j \equiv j_{0} \bmod$ $p^{n+1}$ (with $j \equiv j_{0} \bmod 2^{n+2}$ when $p=2$ ), such that $E[p]$ is irreducible, and such that $j$ has no complex multiplication, is infinite.

For each $j \in H_{\lambda, n}$ let $E$ be the curve given by the Weierstrass equation

$$
E: y^{2}+(j-1728) x y=x^{3}-36(j-1728)^{3} x-(j-1728)^{5}
$$

with $j$-invariant $j(E)=j$ and discriminant $\Delta_{n}=j^{2}(j-1728)^{9}$. Since $j \equiv j_{0} \equiv$ $\lambda \bmod p$, and $\lambda$ was chosen so that $\lambda \not \equiv 0,1728 \bmod p$, it follows that $\Delta \in \mathbb{F}_{p}^{\times}$and, in particular, $\Delta \neq 0$. Thus, $E / \mathbb{Q}$ is an elliptic curve with good reduction at $p$. Since $j(E)=j \equiv \lambda \bmod p$, and $\lambda \in \mathbb{F}_{p}$ is an ordinary $j$-invariant, we conclude that $E$ has ordinary good reduction at $p$. Since $j \in H_{\lambda, n}$, the curve $E$ is not a CM curve. Finally, $j(E)=j \equiv j_{0} \bmod p^{n+1}$ if $p>2$, and $j(E) \equiv j_{0} \bmod 2^{n+2}$ if $p=2$. Since $E[p]$ is an irreducible $\operatorname{Gal}(\overline{\mathbb{Q}} / \mathbb{Q})$-module, then by Theorem 4.1 the decomposition group $D_{n}$ is diagonalizable, hence $I_{n}$ is diagonalizable by Lemma 3.1. Therefore, we have $m \geq n$ in Theorem 3.6, and the ramification index in $\mathbb{Q}\left(E\left[p^{n}\right]\right) / \mathbb{Q}$ is exactly $\varphi\left(p^{n}\right)$, as claimed.

Hence, we have shown the existence of infinitely many non-isomorphic, non$\mathrm{CM}$ curves, as in the statement of the theorem, one for each $j$ in the infinite set $H_{\lambda, n}$.

\section{Examples}

In the following examples we follow the recipe in the proof of Theorem 5.4 to find elliptic curves such that the ramification of $p$ in $\mathbb{Q}\left(E\left[p^{n}\right]\right) / \mathbb{Q}$ is precisely $\varphi\left(p^{n}\right)$.

Example 6.1. Let $p=2$, and let $\lambda=1$. In Example 4.4 we have calculated the canonical lifting of $\lambda$, and it is $j_{0}=-3375$. Now we can take $j_{n, k}=$ $-3375+2^{n+2} \cdot k$, for each $n \geq 1$ and $k \geq 1$, and let $E_{n, k}$ be a curve with $j\left(E_{n, k}\right)=j_{n, k}$ given by a Weierstrass model as in the proof of 5.4 , with discriminant $\Delta_{n, k}=j_{n, k}^{2}\left(j_{n, k}-1728\right)^{9}$. The curve $X_{0}(2)$ is of genus 0 , and the function 
$\phi_{2}(h)=(h+16)^{3} / h($ see [14], Section 9$)$. Let us define

$$
f_{2}(j, x)=(x+16)^{3}-j \cdot x=x^{3}+28 x^{2}+(768-j) x+4096 .
$$

It follows that if $f_{2}\left(j_{n, k}, x\right) \in \mathbb{Q}[x]$ has no rational roots $x \in \mathbb{Q}$, then $E_{n, k}[2]$ is irreducible, and therefore the ramification of any prime ideal above 2 in $\mathbb{Q}\left(E_{n, k}\left[2^{n}\right]\right) / \mathbb{Q}$ will be precisely $\varphi\left(2^{n}\right)$. Using the software Magma, we have verified that, indeed, $f_{2}\left(j_{n, k}, x\right)$ is irreducible over $\mathbb{Q}[x]$ for all $1 \leq n \leq 100$ and all $1 \leq k \leq 100$, and none of the $j$-invariants $j_{n, k}$ in this range has $\mathrm{CM}$.

Example 6.2. Let $p=3$, and let $\lambda=2$. In Example 4.4 we have calculated that the canonical lifting of $\lambda$ is $j_{0}=8000$. Now we can take $j_{n, k}=8000+3^{n+1} \cdot k$, for each $n \geq 1$ and $k \geq 1$, and let $E_{n, k}$ be a curve with $j\left(E_{n, k}\right)=j_{n, k}$, and discriminant $\Delta_{n, k}=j_{n, k}^{2}\left(j_{n, k}-1728\right)^{9}$. The curve $X_{0}(3)$ is of genus 0 , and the function $\phi_{3}(h)=(h+27)(h+3)^{3} / h$. Let us define

$$
f_{3}(j, x)=(x+27)(x+3)^{3}-j \cdot x .
$$

It follows that if $f_{3}\left(j_{n, k}, x\right) \in \mathbb{Q}[x]$ has no rational roots $x \in \mathbb{Q}$, then $E_{n, k}[3]$ is irreducible, and therefore the ramification of any prime ideal above 3 in $\mathbb{Q}\left(E_{n, k}\left[3^{n}\right]\right) / \mathbb{Q}$ will be precisely $\varphi\left(3^{n}\right)$. Using the software Magma, we have verified that, indeed, $f_{3}\left(j_{n, k}, x\right)$ is irreducible over $\mathbb{Q}[x]$ for all $1 \leq n \leq 100$ and all $1 \leq k \leq 100$, and none of the $j$-invariants in this range has $\mathrm{CM}$.

Example 6.3. Let $p=5$, and let $\lambda=2$. In Example 4.4 we have calculated that the canonical lifting of $\lambda$ is $j_{0}=-32768$. Now we can take $j_{n, k}=-32768+5^{n+1} \cdot k$, for each $n \geq 1$ and $k \geq 1$, and let $E_{n, k}$ be a curve with $j\left(E_{n, k}\right)=j_{n, k}$, and discriminant $\Delta_{n, k}=j_{n, k}^{2}\left(j_{n, k}-1728\right)^{9}$. The curve $X_{0}(5)$ is of genus 0 , and the function $\phi_{5}(h)=\left(h^{2}+10 h+5\right)^{3} / h$. Let us define

$$
f_{5}(j, x)=\left(x^{2}+10 x+5\right)^{3}-j \cdot x .
$$

It follows that if $f_{5}\left(j_{n, k}, x\right) \in \mathbb{Q}[x]$ has no rational roots $x \in \mathbb{Q}$, then $E_{n, k}[5]$ is irreducible, and therefore the ramification of any prime ideal above 5 in $\mathbb{Q}\left(E_{n, k}\left[5^{n}\right]\right) / \mathbb{Q}$ will be precisely $\varphi\left(5^{n}\right)$. Using the software Magma, we have verified that, indeed, $f_{5}\left(j_{n, k}, x\right)$ is irreducible over $\mathbb{Q}[x]$ for all $1 \leq n \leq 100$ and all $1 \leq k \leq 100$, and none of the $j$-invariants in this range has $\mathrm{CM}$.

Example 6.4. Let $p=37$, and let $\lambda=7$. In Example 4.5 we have calculated that the canonical lifting of $\lambda$ is $j_{0} \in \mathbb{Q}_{37}$, with the following 37-adic expansion:

$$
j_{0}=\left(7,266,11218,1632114 \bmod 37^{4}, 12877080 \bmod 37^{5}, \ldots\right) \in \mathbb{Q}_{37} .
$$

Let $\alpha_{n}$ be a positive integer congruent to $j_{0} \bmod 37^{n+1}$, e.g., $\alpha_{1}=266, \alpha_{2}=11218$, $\alpha_{3}=1632114$, etc. Now we take $j_{n, k}=\alpha_{n}+37^{n+1} k$, for each $n \geq 1$ and $k \geq 1$, and let $E_{n, k}$ be a curve with $j\left(E_{n, k}\right)=j_{n, k}$, and discriminant $\Delta_{n, k}=j_{n, k}^{2}\left(j_{n, k}-1728\right)^{9}$. The curve $X_{0}(37)$ is of genus 2 , and it has only two rational non-cuspidal points, which correspond to the $j$-invariants $j_{1}=-7 \cdot 11^{3}$ and $j_{2}=-7 \cdot 137^{3} \cdot 2083^{3}$. 
It follows that if $j_{n, k} \neq j_{1}$ or $j_{2}$, then $E_{n, k}[37]$ is irreducible, and therefore the ramification of any prime ideal above 37 in $\mathbb{Q}\left(E_{n, k}\left[37^{n}\right]\right) / \mathbb{Q}$ will be precisely $\varphi\left(37^{n}\right)$. Since $j_{n, k}$ is always positive, and $j_{1}, j_{2}<0$, it follows that $j_{n, k} \neq j_{1}$ or $j_{2}$, for all $k \geq 0$, and all $n \geq 1$, and the ramification properties we seek actually hold for all curves $E_{n, k}$. Moreover, the only positive CM $j$-invariants are $\equiv 0,6,8,10,26,33 \bmod 37$. Since none of them is $\equiv 7 \bmod 37$, we conclude that none of the $j_{n, k}$ have complex multiplication.

\section{7. $\mathrm{SL}_{2}$ extensions of cyclotomic fields}

In this section we are interested to construct examples of $\operatorname{SL}\left(2, \mathbb{Z} / p^{n} \mathbb{Z}\right)$ extensions of $\mathbb{Q}\left(\zeta_{p^{n}}\right)$, that are unramified at primes above $p$.

Example 7.1. Let $p=37$, as in the previous example, and consider the curve

$$
E: y^{2}=x^{3}+x^{2}+17317393168 x-2056380789861728
$$

with $j$-invariant $j(E)=266$ and ordinary good reduction at $p=37$. Since 266 is not one of 13 rational CM $j$-invariants (see [25], Appendix A, §3), it follows that $E$ is not a CM curve. Since $j(E)=266$ is not one of two rational noncuspidal points of $X_{0}(37)$, it follows that $E[37]$ is irreducible as a Galois module. Hence, Theorem 4.1 and Theorem 3.6 show that the ramification of any prime ideal above 37 in $\mathbb{Q}(E[37]) / \mathbb{Q}$ is precisely $\varphi(37)=36$. Since $\mathbb{Q}\left(\zeta_{37}\right) \subset \mathbb{Q}(E[37])$, it follows that $\mathbb{Q}(E[37]) / \mathbb{Q}\left(\zeta_{37}\right)$ is unramified at all the prime ideals above 37 .

Let $\rho_{E, 37}: \operatorname{Gal}(\overline{\mathbb{Q}} / \mathbb{Q}) \rightarrow \operatorname{GL}(2, \mathbb{Z} / 37 \mathbb{Z})$ be the Galois representation associated to the natural action of Galois on $E[37]$. Using Proposition 19 of [18], one can verify that, in fact, $\rho_{E, 37}$ is surjective (Serre's criterion is also implemented in the software package Sage). Hence, $\operatorname{Gal}(\mathbb{Q}(E[37]) / \mathbb{Q}) \cong \operatorname{GL}(2, \mathbb{Z} / 37 \mathbb{Z})$, and $\operatorname{Gal}\left(\mathbb{Q}(E[37]) / \mathbb{Q}\left(\zeta_{37}\right)\right) \cong \mathrm{SL}(2, \mathbb{Z} / 37 \mathbb{Z})$, because the determinant of $\rho_{E, 37}$ is $\chi$, the cyclotomic character. Hence, $\mathbb{Q}(E[37])$ is a Galois extension of $\mathbb{Q}\left(\zeta_{37}\right)$, with Galois group $\operatorname{SL}(2, \mathbb{Z} / 37 \mathbb{Z})$, and unramified at the prime ideal above 37 .

Notice, however, that the conductor of $E$ is $N_{E}=2^{3} \cdot 7^{2} \cdot 17^{2} \cdot 19^{2} \cdot 43^{2}$. By the criterion of Néron, Ogg, and Shafarevich, the extension $\mathbb{Q}(E[37]) / \mathbb{Q}\left(\zeta_{37}\right)$ may be ramified at primes above 2, 7, 17, 19, and 43 .

Theorem 7.2 (Serre, [18], $\S 2 ;[20]$, Lemme 18; Mazur, [16]). Let $E / \mathbb{Q}$ be an elliptic curve. Let $G$ be the image of $\rho_{E, p}$, and suppose $G \neq \mathrm{GL}(E[p])$. Then one of the following possibilities holds:

(1) $G$ is contained in the normalizer of a split Cartan subgroup of $\mathrm{GL}(E[p])$; or

(2) $G$ is contained in the normalizer of a non-split Cartan subgroup of GL(E[p]); or

(3) the projective image of $G$ in $\mathrm{PGL}(E[p])$ is isomorphic to $A_{4}, S_{4}$ or $A_{5}$, where $S_{n}$ is the symmetric group and $A_{n}$ the alternating group; or

(4) $G$ is contained in a Borel subgroup of $\mathrm{GL}(E[p])$.

Moreover, option (3) can only happen for $p \leq 13$, and option (4) can only happen for $p \leq 163$. 
Mazur [16] has shown that option (4) can only happen if $p \leq 163$, and $p \leq 37$ if $E$ does not have CM. Building on [1] and some recent work of Gaudron and Rémond [7], the collaborators Bilu, Parent and Rebolledo [2] have shown the following result on curves whose image is of split Cartan type.

Theorem 7.3 (Bilu, Parent, Rebolledo, [2]). Let $p \geq 11$, with $p \neq 13$, be a prime number. If $E / \mathbb{Q}$ is an elliptic curve such that the image of $\rho_{E, p}$ is contained in a normalizer of a split Cartan subgroup, then the curve $E / \mathbb{Q}$ has $C M$ by a quadratic imaginary field $K$ and $p$ splits in $K / \mathbb{Q}$.

As a corollary of the two previous theorems, and our Theorem 5.4, we obtain infinitely many examples of the $\mathrm{SL}_{2}$ extensions we want.

Theorem 7.4. For every prime $p \geq 17$ and every $n \geq 1$, and for every ordinary $j$-invariant $\lambda \in \mathbb{F}_{p}$, with $\lambda \not \equiv 0,1728 \bmod p$, there are infinitely many nonisomorphic, non-CM, elliptic curves $E$, defined over $\mathbb{Q}$, with $j(E) \equiv \lambda \bmod p$ (and with ordinary good reduction at $p$ ) such that the ramification index of $p$ in the extension $\mathbb{Q}\left(E\left[p^{n}\right]\right) / \mathbb{Q}$ is exactly $\varphi\left(p^{n}\right)$, and $\operatorname{Gal}\left(\mathbb{Q}\left(E\left[p^{n}\right]\right) / \mathbb{Q}\left(\zeta_{p^{n}}\right)\right) \cong \operatorname{SL}\left(2, \mathbb{Z} / p^{n} \mathbb{Z}\right)$. In particular, $\mathbb{Q}\left(E\left[p^{n}\right]\right) / \mathbb{Q}\left(\zeta_{p^{n}}\right)$ is a $\mathrm{SL}\left(2, \mathbb{Z} / p^{n} \mathbb{Z}\right)$ extension, unramified at primes above $p$.

Proof. Let $p \geq 17$ be a prime, let $n \geq 1$ be fixed, and let $\lambda \in \mathbb{F}_{p}$ be an ordinary $j$-invariant with $j \neq \equiv 0,1728 \bmod p$. Let $E$ be one of the infinitely many nonisomorphic, non-CM elliptic curves whose existence is proven by Theorem 5.4 , and let $G$ be the image of the representation $\rho_{E, p}: \operatorname{Gal}(\overline{\mathbb{Q}} / \mathbb{Q}) \rightarrow \operatorname{GL}(2, \mathbb{Z} / p \mathbb{Z})$. If $G \neq \mathrm{GL}(2, \mathbb{Z} / p \mathbb{Z})$, then $G$ falls in one of the four possibilities of Theorem 7.2:

(1) If $G$ is contained in the normalizer of a split Cartan subgroup of GL( $E[p])$, and $p \geq 17$, then Theorem 7.3 implies that $E$ is CM. However, $E$ as in Theorem 5.4 is not CM.

(2) Suppose $G$ is contained in the normalizer of a non-split Cartan subgroup of $\operatorname{GL}(E[p])$. This case is impossible, because with respect to a certain basis, $G$ contains the image of $I_{1}(\Omega \mid p)$, the inertia sugroup of $\Omega$ over $p$ in $\operatorname{Gal}(\mathbb{Q}(E[p]) / \mathbb{Q})$, which is a semi-split Cartan subgroup of the form

$$
\left\{\left(\begin{array}{cc}
a & 0 \\
0 & 1
\end{array}\right): a \in(\mathbb{Z} / p \mathbb{Z})^{\times}\right\}
$$

However, a normalizer of a non-split Cartan subgroup cannot contain a semisplit Cartan.

(3) If the projective image of $G$ in $\operatorname{PGL}(E[p])$ is isomorphic to $A_{4}, S_{4}$ or $A_{5}$, then $p \leq 13$, but we have assumed that $p \geq 17$.

(4) If $G$ is contained in a Borel subgroup of $\operatorname{GL}(E[p])$, then $E[p]$ is not irreducible, but the curves $E$ were chosen so that the $p$-torsion was an irreducible Galois module, so that we could apply Gross' criterion. 
Hence, the only possibility is that $G=\mathrm{GL}(2, \mathbb{Z} / p \mathbb{Z})$. Since $p \geq 17 \geq 5$, and our curves are defined over $\mathbb{Q}$, we can use [22], IV-23, Lemma 3 , to conclude that $\rho_{E, p}: \operatorname{Gal}(\overline{\mathbb{Q}} / \mathbb{Q}) \rightarrow \operatorname{GL}\left(2, \mathbb{Z} / p^{n} \mathbb{Z}\right)$ is also surjective (in fact, the representation is surjective $p$-adically). Therefore, $\operatorname{Gal}\left(\mathbb{Q}\left(E\left[p^{n}\right]\right) / \mathbb{Q}\right) \cong \operatorname{GL}\left(2, \mathbb{Z} / p^{n} \mathbb{Z}\right)$, and $\operatorname{Gal}\left(\mathbb{Q}\left(E\left[p^{n}\right]\right) / \mathbb{Q}\left(\zeta_{p^{n}}\right)\right) \cong \operatorname{SL}\left(2, \mathbb{Z} / p^{n} \mathbb{Z}\right)$. Moreover, $\operatorname{Gal}\left(\mathbb{Q}\left(E\left[p^{n}\right]\right) / \mathbb{Q}\left(\zeta_{p^{n}}\right)\right)$ is unramified at primes above $p$ by Theorem 5.4, and this concludes the proof of the theorem.

Example 7.5. For each $n \geq 1$ and each $k \geq 1$, let $E_{n, k} / \mathbb{Q}$ be the elliptic curves described in Example 6.4. Then, these curves are non-isomorphic, non-CM, defined over $\mathbb{Q}$, with $j(E) \equiv 7 \bmod 37$ (and with ordinary good reduction at 37 ) such that the ramification index of 37 in the extension $\mathbb{Q}\left(E\left[37^{n}\right]\right) / \mathbb{Q}$ is exactly $\varphi\left(37^{n}\right)$, and $E[37]$ is an irreducible $\operatorname{Gal}(\overline{\mathbb{Q}} / \mathbb{Q})$-module. Hence, by the same argument as in the proof of Theorem 7.4, we conclude that $\operatorname{Gal}\left(\mathbb{Q}\left(E\left[p^{n}\right]\right) / \mathbb{Q}\left(\zeta_{p^{n}}\right)\right) \cong \mathrm{SL}\left(2, \mathbb{Z} / p^{n} \mathbb{Z}\right)$.

\section{Ramification away from $p$}

The goal of this section is to show that if $E / \mathbb{Q}$ is an elliptic curve such that the Galois representation on the $p$-torsion $\bar{\rho}_{E}: \operatorname{Gal}(\overline{\mathbb{Q}} / \mathbb{Q}) \rightarrow \operatorname{GL}(E[p])$ is absolutely irreducible, then the extension $\mathbb{Q}\left(E\left[p^{n}\right]\right)$ over $\mathbb{Q}\left(\zeta_{p^{n}}\right)$ has to ramify at some nonarchimedian prime away from $p$. Later on, in the last part of this section, we will show examples of elliptic curves where the extension $\mathbb{Q}\left(E\left[p^{n}\right]\right) / \mathbb{Q}\left(\zeta_{p^{n}}\right)$ is ramified above a single prime $q \neq p$. In order to show Theorem 1.2, we shall use Serre's modularity conjecture, which is now a theorem of Khare and Winterberger. Here, however, we only need the so-called level 1 case, which was shown independently by Dieulefait, and Khare.

Theorem 8.1 (Serre's modularity conjecture, [21], [9], [5], [10], [11]). Let $p$ be a prime, let $\mathbb{F}$ be a finite field of characteristic $p$, and let $\bar{\rho}: \operatorname{Gal}(\overline{\mathbb{Q}} / \mathbb{Q}) \rightarrow \operatorname{GL}(2, \mathbb{F})$ be a continuous, absolutely irreducible, two-dimensional, odd (i.e., $\operatorname{det}(\bar{\rho}(\tau))=-1$ for any complex conjugation $\tau)$ Galois representation. Let $k(\bar{\rho})$ be its optimal weight (as defined in [21]) and suppose that $N(\bar{\rho})$, the (prime-to-p) Artin conductor of $\bar{\rho}$, is identically 1 . Then, $\bar{\rho}$ arises from $S_{k(\bar{\rho})}\left(\mathrm{SL}_{2}(\mathbb{Z})\right)$, i.e., there is a cusp form $f \in S_{k(\bar{\rho})}\left(\mathrm{SL}_{2}(\mathbb{Z})\right)$ and an integral model $\rho_{f}: \operatorname{Gal}(\overline{\mathbb{Q}} / \mathbb{Q}) \rightarrow \operatorname{GL}(2, \mathcal{O})$ of its associated $p$-adic Galois representation, with $\mathcal{O}$ the ring of integers of a finite extension of $\mathbb{Q}_{p}$, such that the reduction of $\rho_{f}$ modulo the maximal ideal of $\mathcal{O}$ is isomorphic to $\bar{\rho}$.

Before we prove our theorem, we remind the reader about the definition of the Artin conductor of a representation, following [21], Section 1.2. Let $V$ be a 2dimensional vector space over $\overline{\mathbb{F}}_{p}$ and let $\rho: \operatorname{Gal}(\overline{\mathbb{Q}} / \mathbb{Q}) \rightarrow \operatorname{GL}(V)$ be a continuous Galois representation. Then, the Artin conductor of $\rho$ is defined as

$$
N=\prod_{\ell \neq p} \ell^{n(\ell, \rho)}
$$


where $n(\ell, \rho) \geq 0$ are non-negative integers defined as follows. For each prime number $\ell \neq p$, let $\nu$ be an extension to $\overline{\mathbb{Q}}$ of the $\ell$-adic valuation of $\mathbb{Q}$, and let

$$
G_{0} \supset G_{1} \supset \cdots \supset G_{i} \supset \cdots
$$

be the (higher) ramification groups of $G$ with respect to $\nu$. Let $V_{i}$ be the subspace of $V$ whose elements are fixed by $G_{i}$, and define

$$
n(\ell, p)=\sum_{i=0}^{\infty} \frac{1}{\left[G_{0}: G_{i}\right]} \operatorname{dim} V / V_{i} .
$$

It is worth noting that:

1. $n(\ell, \rho)=0$ if and only if $G_{0}=\{1\}$, i.e., if and only if $\rho$ is unramified at $\ell$, and

2. $n(\ell, \rho)=\operatorname{dim} V / V_{0}$ if and only if $G_{1}=\{1\}$, i.e., if and only if $\rho$ is tamely ramified at $\ell$.

We also need to recall some facts about the optimal weight $k(\bar{\rho})$, which is defined in [21], §2. In particular, we need the following result.

Proposition 8.2 (Serre, [21], §2.9, Prop. 5). Let $p$ be a prime, let $E / \mathbb{Q}_{p}$ be an elliptic curve, and let $\bar{\rho}: \operatorname{Gal}\left(\overline{\mathbb{Q}}_{p} / \mathbb{Q}_{p}\right) \rightarrow \mathrm{GL}\left(2, \mathbb{F}_{p}\right)$ be the representation attached to the natural action of Galois on the p-torsion $E[p]$ of E. Then,

(1) if $E / \mathbb{Q}_{p}$ has good reduction, then $k(\bar{\rho})=2$;

(2) if $E / \mathbb{Q}_{p}$ has multiplicative reduction, then $k(\bar{\rho})=2$ if $\nu_{p}(j(E))$ is divisible by $p$, and $k(\bar{\rho})=p+1$ otherwise.

We are now ready to prove Theorem 1.2. The idea of the proof is due to Robert Pollack.

Proof of Theorem 1.2. Let $E / \mathbb{Q}$ be an elliptic curve such that the Galois representation on the $p$-torsion $\bar{\rho}_{E}: \operatorname{Gal}(\overline{\mathbb{Q}} / \mathbb{Q}) \rightarrow \mathrm{GL}(E[p])$ is absolutely irreducible, and with either good reduction at $p$, or with multiplicative reduction at $p$ and $\nu_{p}(j(E))$ divisible by $p$. Suppose for a contradiction that the extension $\mathbb{Q}\left(E\left[p^{n}\right]\right)$ over $\mathbb{Q}\left(\zeta_{p^{n}}\right)$ is unramified at all primes not above $p$. Then, the extension $\mathbb{Q}(E[p]) / \mathbb{Q}\left(\zeta_{p}\right)$ is also unramified at all primes not above $p$, because of the multiplicativity of ramification indices in towers, and because $\mathbb{Q}\left(\zeta_{p^{n}}\right) / \mathbb{Q}\left(\zeta_{p}\right)$ is only ramified above $p$.

Now, let $\bar{\rho}_{E}: \operatorname{Gal}(\overline{\mathbb{Q}} / \mathbb{Q}) \rightarrow \operatorname{GL}(E[p]) \cong \operatorname{GL}\left(2, \mathbb{F}_{p}\right)$ be the representation associated to the natural Galois action on $E[p]$. This representation is continuous, absolutely irreducible (by assumption), and odd (see, for instance, [17], Section 1.1.2). By our assumptions on the reduction type of $E$ and Proposition 8.2, its weight is $k\left(\bar{\rho}_{E}\right)=2$. Moreover, we have shown that $\mathbb{Q}(E[p]) / \mathbb{Q}$ is only ramified above $p$ and, thus, $\bar{\rho}_{E}$ is unramified outside $p$. It follows from our remarks above on the Artin conductor that $N\left(\bar{\rho}_{E}\right)=1$. Hence, Theorem 8.1 implies that $\bar{\rho}_{E}$ arises from $S_{2}\left(\mathrm{SL}_{2}(\mathbb{Z})\right)$. However, $S_{2}\left(\mathrm{SL}_{2}(\mathbb{Z})\right)=\{0\}$ by Theorem 3.5.2 of [4] so this is impossible. 
We remark that if $\operatorname{Gal}(\mathbb{Q}(E[p]) / \mathbb{Q}) \cong \mathrm{GL}\left(2, \mathbb{F}_{p}\right)$, then the Galois representation $\bar{\rho}_{E}$ is absolutely irreducible, therefore satisfying the hypothesis of Theorem 1.2. It follows that if $E / \mathbb{Q}$ has good reduction at $p$, and $\operatorname{Gal}(\mathbb{Q}(E[p]) / \mathbb{Q}) \cong \mathrm{GL}\left(2, \mathbb{F}_{p}\right)$, then $\mathbb{Q}\left(E\left[p^{n}\right]\right) / \mathbb{Q}\left(\zeta_{p^{n}}\right)$ must ramify (at least) at some prime above a rational prime $q \neq p$. In the rest of this section, we find examples where the ramification happens exactly at primes above one single rational prime $q \neq p$.

Theorem 8.3 (Kida, [12], Theorems 1.1 and 1.2). Let $q$ and $p \geq 2$ be distinct primes. Let $E / \mathbb{Q}$ be an elliptic curve. Then:

(1) The extension $\mathbb{Q}(E[p]) / \mathbb{Q}$ is unramified at the primes above $q$ if and only if $E / \mathbb{Q}$ has (a) good reduction at $q$, or $(b)$ multiplicative reduction at $q$ and $\nu_{p}\left(-\nu_{q}(j(E))\right)$ is a positive integer.

(2) Assume that $\mathbb{Q}(E[p]) / \mathbb{Q}$ is unramified at $q$, and $E / \mathbb{Q}$ has multiplicative reduction at $q$. Then, $\mathbb{Q}\left(E\left[p^{n}\right]\right) / \mathbb{Q}$ is unramified if and only if $1 \leq n \leq$ $\nu_{p}\left(-\nu_{q}(j(E))\right)$.

Example 8.4. Let $E / \mathbb{Q}$ be the curve with Cremona label 11a1. We saw in Question 4.7 that $\mathbb{Q}(E[5])=\mathbb{Q}\left(\zeta_{5}\right)$, and therefore the extension $\mathbb{Q}(E[5]) / \mathbb{Q}\left(\zeta_{5}\right)$ is trivially unramified at 5 . Note however that $E$ has bad reduction at 11 , and $\mathbb{Q}(E[5]) / \mathbb{Q}$ is unramified at 11 . Kida's Theorem 8.3 says that the bad reduction at 11 must be multiplicative, and $\nu_{5}\left(-\nu_{11}(j(E))\right)$ must be positive. Indeed, the reduction is bad multiplicative $\left(\Delta=-11^{5}, c_{4}=2^{4} \cdot 31\right)$ and

$$
j(E)=-\frac{122023936}{161051}=-\frac{2^{12} \cdot 31^{3}}{11^{5}},
$$

and so $\nu_{5}\left(-\nu_{11}(j(E))\right)=1>0$.

Example 8.5. In this example we find primes $p$, integers $n \geq 1$, and elliptic curves $E / \mathbb{Q}$ such that $\operatorname{Gal}\left(\mathbb{Q}\left(E\left[p^{n}\right]\right) / \mathbb{Q}\right) \cong \operatorname{GL}\left(2, \mathbb{Z} / p^{n} \mathbb{Z}\right)$ and such that $\mathbb{Q}\left(E\left[p^{n}\right]\right) / \mathbb{Q}\left(\zeta_{p}^{n}\right)$ is unramified at primes above $p$, and only ramified at primes above at most one prime $q \neq p$. In order to find such examples, it suffices to find elliptic curves with the following properties:

(a) $E / \mathbb{Q}$ with ordinary good reduction at $p$;

(b) if $j(E) \equiv \lambda \in \mathbb{F}_{p}$, and $j_{0}$ is the canonical lift of $\lambda$, then $j(E) \equiv j_{0} \bmod p^{n+1}$ if $p$ is odd (and $\bmod 2^{n+2}$ for $p=2$ );

(c) the representation $\rho_{E, p}: \operatorname{Gal}(\overline{\mathbb{Q}} / \mathbb{Q}) \rightarrow \operatorname{GL}\left(2, \mathbb{Z} / p^{n} \mathbb{Z}\right)$ must be surjective (one can be verify with Sage whether $\bar{\rho}_{E, p}: \operatorname{Gal}(\overline{\mathbb{Q}} / \mathbb{Q}) \rightarrow \operatorname{GL}\left(2, \mathbb{F}_{p}\right)$ is surjective; if $p \geq 5$, then $\bar{\rho}_{E, p}$ is surjective if and only if $\rho_{E, p}$ is surjective);

(d) there is at most one prime of additive reduction $q$; and (e) every prime $\ell$ of multiplicative reduction satisfies $1 \leq n \leq \nu_{p}\left(-\nu_{\ell}(j(E))\right)$.

For instance, let $p=5$, and let $E / \mathbb{Q}$ be the curve with Cremona reference "61a1" and $j$-invariant $j=-912673 / 61$, given by the model

$$
y^{2}+x y=x^{3}-2 x+1 .
$$

The curve $E / \mathbb{Q}$ has bad multiplicative reduction at 61 (with Kodaira symbol I1), and good reduction elsewhere. Note that $j \equiv 7 \equiv-32768 \equiv j_{0} \bmod 25$, where 
$j_{0}=-32768$ is the canonical lift of $\lambda=2 \in \mathbb{F}_{5}$. With the help of Sage, we have verified that $\rho_{E, 5}: \operatorname{Gal}(\overline{\mathbb{Q}} / \mathbb{Q}) \rightarrow \operatorname{GL}\left(2, \mathbb{F}_{5}\right)$ is surjective. Finally, notice that there is only one prime of bad reduction, namely $q=61$. Hence, all the necessary hypotheses are met, and $\mathbb{Q}(E[5]) / \mathbb{Q}\left(\zeta_{5}\right)$ is a $\operatorname{SL}\left(2, \mathbb{F}_{5}\right)$ extension that is only ramified at primes above a unique rational prime, namely $q=61$.

In the following table we give a few examples we have found using Cremona's tables (all curves with conductor $\leq 300000$, a total of 1887909 curves) of primes $p$, integers $n \geq 1$, and curves $E / \mathbb{Q}$ with $j$-invariant $j(E)$ that verify conditions (a) through (e) as above. In all examples we have verified that $\bar{\rho}_{E, p}: \operatorname{Gal}(\overline{\mathbb{Q}} / \mathbb{Q}) \rightarrow$ $\operatorname{GL}\left(2, \mathbb{F}_{p}\right)$ is surjective (with Sage). If $p \geq 5$, then the Galois representation $\rho_{E, p}$ : $\operatorname{Gal}(\overline{\mathbb{Q}} / \mathbb{Q}) \rightarrow \operatorname{GL}\left(2, \mathbb{Z} / p^{n} \mathbb{Z}\right)$ is also surjective. In all cases, the Kodaira symbol at $q$ is $\mathrm{I} 1$.

\begin{tabular}{c|c|c|c|c|c}
\hline$p$ & $n$ & $j_{0}$ & $j(E)$ & Cremona & $q$ \\
\hline 2 & 8 & -3375 & $-185193 / 114407$ & $114407 \mathrm{a} 1$ & 114407 \\
3 & 6 & -32768 & $-5168743489 / 143729$ & $143729 \mathrm{a} 1$ & 143729 \\
5 & 5 & -32768 & $-147197952 / 2539$ & $2539 \mathrm{a} 1$ & 2539 \\
7 & 4 & $2126+O\left(7^{5}\right)$ & $38272753 / 21283$ & $21283 \mathrm{a} 1$ & 21283 \\
11 & 3 & $7665+O\left(11^{5}\right)$ & $65597103937 / 110879$ & $110879 \mathrm{c} 1$ & 110879 \\
13 & 2 & -884736000 & $-35937 / 1873$ & $1873 \mathrm{a} 1$ & 1873 \\
17 & 2 & $74802+O\left(17^{5}\right)$ & $-117649 / 89$ & $89 \mathrm{a} 1$ & 89 \\
19 & 3 & $-752904+O\left(19^{5}\right)$ & $49836032 / 57587$ & $57587 \mathrm{a} 1$ & 57587 \\
\hline
\end{tabular}

We conclude with an example of an elliptic curve whose conductor is not prime. Let $E / \mathbb{Q}$ be the curve with label "309a1" and model

$$
y^{2}+x y=x^{3}-6 x+9
$$

The curve $E / \mathbb{Q}$ has bad multiplicative reduction at 3 and 103 , with Kodaira symbols I5 and I1 respectively, and good reduction elsewhere. The $j$-invariant of $E$ satisfies

$$
j(E)=-\frac{24137569}{25029}=-17^{6} \cdot 3^{-5} \cdot 103^{-1} \equiv 14 \bmod 25 .
$$

Thus, $j(E) \equiv j_{0} \bmod 25$, where the canonical invariant in this case is $j_{0}=$ -884736 (see Example 4.4). With the help of Sage, we have verified that $\rho_{E, 5}$ : $\operatorname{Gal}(\overline{\mathbb{Q}} / \mathbb{Q}) \rightarrow \operatorname{GL}\left(2, \mathbb{F}_{5}\right)$ is surjective. Hence, all the necessary hypotheses are met, and $\mathbb{Q}(E[5]) / \mathbb{Q}\left(\zeta_{5}\right)$ is a $\mathrm{SL}\left(2, \mathbb{F}_{5}\right)$ extension that is only ramified at primes above a unique rational prime, namely $q=103$. However, the extension $\mathbb{Q}(E[25]) / \mathbb{Q}\left(\zeta_{25}\right)$ also ramifies at primes above 3 .

\section{References}

[1] Bilu, Y. And Parent, P.: Serre's uniformity problem in the split Cartan case. Ann. of Math. (2) 173 (2011), no. 1, 569-584.

[2] Bilu, Y., Parent, P. and Rebolledo, M.: Rational points on $X_{0}^{+}\left(p^{r}\right)$. Ann. Inst. Fourier (Grenoble) 63 (2013), no. 3, 957-984. 
[3] Conrad, B. And Rubin, K.: Arithmetic algebraic geometry. IAS/Park City Mathematics Series 9, American Mathematical Society, Providence, RI, 2001.

[4] Diamond, F. And Shurman, J.: A first course in modular forms. Graduate Texts in Mathematics 228, Springer-Verlag, New York, 2005.

[5] Dieulefait, L.: The level 1 weight 2 case of Serre's conjecture. Rev. Mat. Iberoam. 23 (2007), no. 3, 1115-1124.

[6] Gross, B. H.: A tameness criterion for Galois representations associated to modular forms $(\bmod p)$. Duke Math. J. 61 (1990), no. 2, 445-517.

[7] Gaudron, É. And RÉmond, G.: Théorème des périodes et degrés minimaux d'isogénies. Comment. Math. Helv. 89 (2014), no. 2, 343-403.

[8] Iwasawa, K.: Local class field theory. Oxford Science Publications, Oxford University Press, New York, 1986.

[9] Khare, C.: Serre's modularity conjecture: the level one case. Duke Math. J. 134 (2006), no. 3, 557-589.

[10] Khare, C. and Wintenberger, J-P.: Serre's modularity conjecture. I. Invent. Math. 178 (2009), no. 3, 485-504.

[11] Khare, C. And Wintenberger, J-P.: Serre's modularity conjecture. II. Invent. Math. 178 (2009), no. 3, 505-586.

[12] KidA, M.: Ramification in the division fields of an elliptic curve. Abh. Math. Sem. Univ. Hamburg 73 (2003), 195-207.

[13] Lang, S.: Fundamentals of diophantine geometry. Springer-Verlag, New York, 1983.

[14] Lozano-Robledo, Á.: On the field of definition of $p$-torsion points on elliptic curves over the rationals. Math. Ann. 357 (2013), no. 1, 279-305.

[15] Lubin, J., Serre, J-P. And Tate, J.: Elliptic curves and formal groups. Lecture notes prepared in connection with the seminars held at the Summer Institute on Algebraic Geometry, Whitney Estate, Woods Hole, Massachusetts, July 6-31, 1964.

[16] Mazur, B.: Rational isogenies of prime degree. Invent. Math. 44 (1978), no. 2, 129-162.

[17] Ribet, K. A. And Stein, W. A.: Lectures on Serre's conjectures. In Arithmetic algebraic geometry (Park City, UT, 1999), 143-232. IAS/Park City Math. Ser. 9, Amer. Math. Soc., Providence, RI, 2001.

[18] Serre, J.-P.: Propriétés galoisiennes des points d'ordre fini des courbes elliptiques. Invent. Math. 15 (1972), no. 4, 259-331.

[19] Serre, J.-P. And Zagier, D. B.: Modular functions of one variable V. Lecture Notes in Mathematics 601, Springer-Verlag, Berlin-New York, 1977.

[20] Serre, J.-P.: Quelques applications du théorème de densité de Chebotarev. Inst. Hautes Études Sci. Publ. Math. 54 (1981), 323-401.

[21] Serre, J.-P.: Sur les représentations modulaires de degré 2 de $\operatorname{Gal}(\overline{\mathbb{Q}} / \mathbb{Q})$. Duke Math. J. 54 (1987), no. 1, 179-230.

[22] Serre, J.-P.: Abelian l-adic representations and elliptic curves., Research Notes in Mathematics 7, A K Peters, Wellesley, MA, 1998.

[23] Serre, J-P.: Topics in Galois theory. Research Notes in Mathematics 1, A K Peters, Wellesley, MA, 2008.

[24] Silverman, J. H.: The arithmetic of elliptic curves. Graduate Texts in Mathematics 106, Springer, Dordrecht, 2009. 
[25] Silverman, J.H.: Advanced topics in the arithmetic of elliptic curves. Graduate Texts in Mathematics 151, Springer-Verlag, New York, 1994

Received December 30, 2013; revised November 28, 2014.

Álvaro Lozano-Robledo: Department of Mathematics, University of Connecticut, Storrs, CT 06269, USA.

E-mail: alvaro.lozano-robledo@uconn.edu 